\title{
Surface wave propagation in a fluid-saturated incompressible porous medium
}

\author{
RAJNEESH KUMAR ${ }^{1}$ and B S HUNDAL ${ }^{2}$ \\ ${ }^{1}$ Department of Mathematics, Kurukshetra University, Kurukshetra, Haryana \\ ${ }^{2}$ Department of Mathematics, S. R. Government College for Women, Amritsar, \\ Punjab \\ e-mail: hundal_bs@yahoo.co.in; rajneesh_kuk@rediffmail.com
}

MS received 31 January 2006; revised 26 March 2007

\begin{abstract}
A study of surface wave propagation in a fluid-saturated incompressible porous half-space lying under a uniform layer of liquid is presented. The dispersion relation connecting the phase velocity with wave number is derived. The variation of phase velocity and attenuation coefficients with wave number is presented graphically and discussed. As a particular case, the propagation of Rayleigh type surface waves at the free surface of an incompressible porous half-space is also deduced and discussed.
\end{abstract}

Keywords. Incompressible porous medium; volume fractions; frequency equation; phase velocity; wave number; attenuation coefficient.

\section{Introduction}

Porous media theories play an important role in many branches of engineering including materials science, petroleum industry, chemical engineering, biomechanics, soil mechanics and other such fields of engineering. Most of the modern engineering structures are generally made up of multiphase porous continuum and the classical theory, which represents a fluidsaturated porous medium as a single-phase material, is inadequate to study the mechanical behaviour of such materials especially when the pores are filled with liquid. In this case the solid and liquid phases have different motions. Due to these different motions, the different material properties and the complicated geometry of pore-structure; the mechanical behaviour of a fluid-saturated porous medium becomes more difficult. So researchers from time to time have tried to overcome this difficulty and a considerable work is available in the literature. For more detailed and for the historical review on the subject of multiphase continuum mechanics, the reader is referred to the work of de Boer \& Ehlers (1988) or recently published monograph de Boer (2000).

Based on the work of von Terzaghi $(1923,1925)$, Biot (1941) proposed a general theory of three-dimensional deformations of fluid-saturated porous elastic solids. Subsequently, Biot $(1956 a, b, 1962)$ did the dynamic extension of his theory and proposed the propagation of two dilatational and one rotational elastic waves in fluid-saturated porous solids. Biot theory is 
based on the assumption of compressible constituents and till recently, some of his results have been taken as standard references and basis for subsequent analysis in acoustic, geophysics and other related fields. Different authors have investigated the relating problems by considering different theories of wave propagation (Levy 1979, Auriault 1980, Prevost 1982, Zienkiewicz \& Shiomi 1984).

Based on the Fillunger model (1933), (which is further based on the concept of volume fractions combined with surface porosity coefficients), another interesting theory in which all the constituents of a porous medium are assumed to be incompressible was developed and used by (Bowen 1980, de Boer \& Ehlers 1990, Ehlers 1993). There are reasonable grounds to assume that the constituents of many fluid-saturated porous media are incompressible. For example, taking the composition of soil; solid constituents are incompressible and liquid constituents which are generally water or oils are also incompressible. Moreover, in an empty porous solid as a case of classical theory, the change in volume is due to the change in porosity during the propagation of a longitudinal wave. The assumption of incompressible constituents does not only meet the properties appearing in many branches of engineering practice, but it avoids the introduction of many complicated material parameters as considered in the Biot theory. So, this model meets the assumptions and requirements of further scientific developments. Based on this theory de Boer \& Ehlers (1993) and recently Kumar \& Hundal $(2002,2003,2004 a, b)$ have studied the problems of wave propagation in fluid-saturated incompressible porous media.

Many studies have discussed the surface wave propagation in elastic media and a comprehensive review is available in the standard texts, e.g., Ewing et al (1957) and Achenbach (1976). The surface waves discussed in these texts are within the scope of single-phase models, but the presence of fluid in the pores of an elastic porous solid might have affected the motion of solid particles. As far as the multi-phase systems are concerned; there is considerable work concerning the surface wave propagation in fluid-saturated porous media at the present time, and a brief review is available in (Kumar \& Miglani 1996, Kumar \& Deswal 1966, Liu \& Liu 2004, Edelman 2004). But all this is based on the classical Biot's model where the constituents of a fluid-saturated porous medium are assumed to be compressible.

In this paper, propagation of surface waves in a half-space of a fluid-saturated incompressible porous medium lying under a uniform layer of a liquid is investigated. The fluid-saturated porous material is modelled as a two-phase system composed of incompressible solid and fluid phases. Frequency equation relating the phase velocity with the wave number is derived. As a particular case, the propagation of Rayleigh type surface waves at the free surface of an incompressible porous half-space is also discussed and equations of trajectories of medium particles are derived.

\section{Basic equations}

Within the framework of modern porous media theories de Boer \& Ehlers (1990), the equations governing the deformation of an incompressible porous medium are:

$$
\begin{aligned}
& \operatorname{div} .\left(\eta^{S} \dot{\boldsymbol{x}}_{\boldsymbol{S}}+\eta^{F} \dot{\boldsymbol{x}}_{\boldsymbol{F}}\right)=0, \\
& \operatorname{div} . \boldsymbol{T}_{\boldsymbol{E}}^{\boldsymbol{S}}-\eta^{S} \text { grad.p }+\rho^{S}\left(\boldsymbol{b}-\ddot{\boldsymbol{x}}_{\boldsymbol{S}}\right)-\boldsymbol{P}_{\boldsymbol{E}}^{\boldsymbol{F}}=0, \\
& \operatorname{div} . \boldsymbol{T}_{\boldsymbol{E}}^{\boldsymbol{F}}-\eta^{F} \text { grad.p }+\rho^{F}\left(\boldsymbol{b}-\ddot{\boldsymbol{x}}_{\boldsymbol{F}}\right)+\boldsymbol{P}_{\boldsymbol{E}}^{\boldsymbol{F}}=0,
\end{aligned}
$$


where $\dot{\boldsymbol{x}}_{\boldsymbol{i}}$ and $\ddot{\boldsymbol{x}}_{\boldsymbol{i}}(i=F, S)$ denote the velocities and accelerations of solid and fluid phases respectively and $p$ is the effective pore pressure of the incompressible pore fluid. $\rho^{S}$ and $\rho^{F}$ are the densities of the solid and fluid phases respectively and $\boldsymbol{b}$ is the body force per unit volume. $\boldsymbol{T}_{\boldsymbol{E}}^{\boldsymbol{S}}, \boldsymbol{T}_{\boldsymbol{E}}^{\boldsymbol{F}}$ and $\boldsymbol{P}_{\boldsymbol{E}}^{\boldsymbol{F}}$ are called the extra quantities for which the constitutive equations must be formulated and $\eta^{S}, \eta^{F}$ are the volume fractions satisfying

$$
\eta^{S}+\eta^{F}=1 \text {. }
$$

If $\boldsymbol{u}_{\boldsymbol{S}}$ and $\boldsymbol{u}_{\boldsymbol{F}}$ are the displacement vectors for solid and fluid phases then

$$
\dot{x}_{S}=\dot{u}_{S}, \ddot{x}_{S}=\ddot{u}_{S}, \dot{x}_{F}=\dot{u}_{F}, \ddot{x}_{F}=\ddot{u}_{F} .
$$

The investigations to follow are restricted to a linear isotropic, elastic porous medium filled with an inviscid liquid. So the constitutive equations for extra stresses and extra momentum are given by de Boer et al (1993) as

$$
\begin{aligned}
& \boldsymbol{T}_{\boldsymbol{E}}^{\boldsymbol{S}}=2 \mu^{S} \boldsymbol{E}_{\boldsymbol{S}}+\lambda^{S}\left(\boldsymbol{E}_{\boldsymbol{S} . \boldsymbol{I}}\right) I, \\
& \boldsymbol{T}_{\boldsymbol{E}}^{\boldsymbol{F}}=\mathbf{0}, \\
& \boldsymbol{P}_{\boldsymbol{E}}^{\boldsymbol{F}}=-\boldsymbol{S}_{\boldsymbol{V}}\left(\dot{\boldsymbol{u}}_{\boldsymbol{F}}-\dot{\boldsymbol{u}}_{\boldsymbol{S}}\right),
\end{aligned}
$$

where $\lambda^{S}$ and $\mu^{S}$ are the macroscopic Lame's parameters of the porous solid and $\boldsymbol{E}_{\boldsymbol{S}}$ is the linearised Langrangian strain tensor defined as

$$
\boldsymbol{E}_{\boldsymbol{S}}=\frac{1}{2}\left(\operatorname{grad} \boldsymbol{u}_{\boldsymbol{S}}+\operatorname{grad}^{T} \boldsymbol{u}_{\boldsymbol{S}}\right) .
$$

In the case of isotropic permeability, the tensor $S_{V}$ describing the coupled interaction between the solid and fluid is given by de Boer \& Ehlers $(1990,1993)$ as

$$
\boldsymbol{S}_{\boldsymbol{V}}=\frac{\left(\eta^{F}\right)^{2} \gamma^{F R}}{K^{F}} \boldsymbol{I}
$$

where $\gamma^{F R}$ is the effective specific weight of the fluid and $K^{F}$ is the Darcy's permeability coefficient of the porous medium.

\section{Formulation of the problem and its solution}

Consider a half-space of a fluid-saturated incompressible porous medium lying under a uniform layer of liquid of thickness $H$. The motion of the materials is considered in $x-z$ plane, where $x$-axis is taken along the interface, and the $z$-axis is taken along the direction of increasing depth. Hence the fluid-saturated incompressible porous medium occupies the region $z>0$, whereas the region $-H<z<0$ is occupied by the liquid layer with $z=0$ as the interface between the liquid layer and the half-space as shown in figure 1. Also, in the scope of infinitesimal deformations, all the terms of higher orders are neglected. Moreover, the small variation in volume fractions is also neglected. So taking the body forces to be absent and after some simplification the equations (1) to (8) for the half-space of a fluid-saturated incompressible medium can be expressed in the following form:

$$
\left(\lambda^{S}+\mu^{S}\right) \frac{\partial \theta^{S}}{\partial x}+\mu^{S} \nabla^{2} u^{S}-\eta^{S} \frac{\partial p}{\partial x}-\rho^{S} \frac{\partial^{2} u^{S}}{\partial t^{2}}+S_{V}\left(\frac{\partial u^{F}}{\partial t}-\frac{\partial u^{S}}{\partial t}\right)=0,
$$




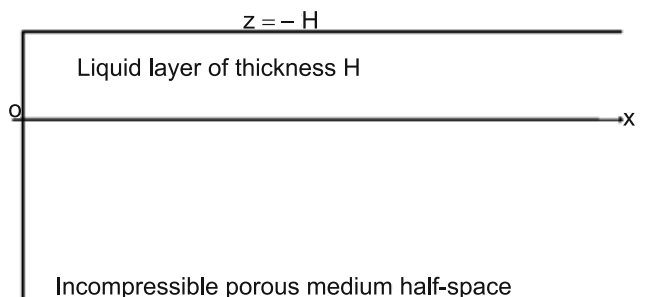

Incompressible porous medium half-space

Figure 1. Geometry of the investigated problem.

$$
\begin{aligned}
& \left(\lambda^{S}+\mu^{S}\right) \frac{\partial \theta^{S}}{\partial z}+\mu^{S} \nabla^{2} w^{S}-\eta^{S} \frac{\partial p}{\partial z}-\rho^{S} \frac{\partial^{2} w^{S}}{\partial t^{2}}+S_{V}\left(\frac{\partial w^{F}}{\partial t}-\frac{\partial w^{S}}{\partial t}\right)=0 \\
& \eta^{F} \frac{\partial p}{\partial x}+\rho^{F} \frac{\partial^{2} u^{F}}{\partial t^{2}}+S_{V}\left(\frac{\partial u^{F}}{\partial t}-\frac{\partial u^{S}}{\partial t}\right)=0 \\
& \eta^{F} \frac{\partial p}{\partial z}+\rho^{F} \frac{\partial^{2} w^{F}}{\partial t^{2}}+S_{V}\left(\frac{\partial w^{F}}{\partial t}-\frac{\partial w^{S}}{\partial t}\right)=0 \\
& \eta^{S}\left(\frac{\partial^{2} u^{S}}{\partial x \partial t}+\frac{\partial^{2} w^{S}}{\partial z \partial t}\right)+\eta^{F}\left(\frac{\partial^{2} u^{F}}{\partial x \partial t}+\frac{\partial^{2} w^{F}}{\partial z \partial t}\right)=0
\end{aligned}
$$

where

$$
\theta^{S}=\frac{\partial u^{S}}{\partial x}+\frac{\partial w^{S}}{\partial z} .
$$

For the layer, equations governing the motion of a liquid are given by Ewing et al (1957) as

$$
\begin{aligned}
& \lambda^{L} \nabla\left(\nabla \boldsymbol{u}^{\boldsymbol{L}}\right)=\rho^{L} \frac{\partial^{2} \boldsymbol{u}^{\boldsymbol{L}}}{\partial t^{2}}, \\
& \tau_{m n}^{L}=\lambda^{L} \nabla \boldsymbol{u}^{\boldsymbol{L}} \delta_{m n},, m, n=1,2,3 .
\end{aligned}
$$

In these two equations $\boldsymbol{u}^{L}$ is the displacement vector, $\lambda^{L}$ is the bulk modulus of the liquid $\rho^{L}$ is its density and $\tau_{m n}^{L}$ are the components of the stress in the liquid. For the present problem, the displacement vector $\boldsymbol{u}^{L}=\left(u^{L}, 0, w^{L}\right)$, so (17) and (18) are simplified as

$$
\lambda^{L}\left(\frac{\partial^{2} u^{L}}{\partial x^{2}}+\frac{\partial^{2} w^{L}}{\partial x \partial z}\right)=\rho^{L} \frac{\partial^{2} u^{L}}{\partial t^{2}},
$$




$$
\begin{aligned}
& \lambda^{L}\left(\frac{\partial^{2} u^{L}}{\partial x \partial z}+\frac{\partial^{2} w^{L}}{\partial z^{2}}\right)=\rho^{L} \frac{\partial^{2} w^{L}}{\partial t^{2}}, \\
& \tau_{z z}^{L}=\tau_{x x}^{L}=\lambda^{L}\left(\frac{\partial u^{L}}{\partial x}+\frac{\partial w^{L}}{\partial z}\right) .
\end{aligned}
$$

Again following Ewing et al (1957), for the liquid pressure $p^{L}$ we have:

$$
p^{L}=-\tau_{z z}^{L}
$$

For further considerations, it is convenient to introduce, (11) to (22), the dimensionless quantities defined as:

$$
\begin{aligned}
& x^{\prime}=\frac{\omega^{\prime}}{c_{1}} x, \quad z^{\prime}=\frac{\omega^{\prime}}{c_{1}} z, \quad t^{\prime}=\omega^{\prime} t, \quad u^{\prime S}=\left(\frac{\lambda^{S}+2 \mu^{S}}{E}\right) \frac{\omega^{\prime}}{c_{1}} u^{S}, \\
& w^{\prime S}=\left(\frac{\lambda^{S}+2 \mu^{S}}{E}\right) \frac{\omega^{\prime}}{c_{1}} w^{S}, \quad u^{\prime F}=\left(\frac{\lambda^{S}+2 \mu^{S}}{E}\right) \frac{\omega^{\prime}}{c_{1}} u^{F}, \\
& {w^{\prime} F}^{F}=\left(\frac{\lambda^{S}+2 \mu^{S}}{E}\right) \frac{\omega^{\prime}}{c_{1}} u^{F}, \quad p^{\prime}=\frac{p}{E}, \quad \tau_{x z}^{\prime}=\frac{\tau_{x z}}{E}, \quad \tau_{z z}^{\prime}=\frac{\tau_{z z}}{E} \\
& u^{\prime L}=\left(\frac{\lambda^{S}+2 \mu^{S}}{E}\right) \frac{\omega^{\prime}}{c_{1}} u^{L}, \quad w^{\prime L}=\left(\frac{\lambda^{S}+2 \mu^{S}}{E}\right) \frac{\omega^{\prime}}{c_{1}} u^{L}, \\
& p^{\prime L}=\frac{p^{L}}{E}, \quad \tau_{z z}^{\prime L}=\frac{\tau_{z z}^{L}}{E} .
\end{aligned}
$$

In these relations $E$ is the Young's modulus of the solid phase, $\omega^{\prime}$ the characteristic frequency of the medium, and $c_{1}$ is the velocity of a longitudinal wave propagating in a fluid-saturated incompressible porous medium (de Boer et al 1993) and is given by

$$
c_{1}=\sqrt{\frac{\left(\eta^{F}\right)^{2}\left(\lambda^{S}+2 \mu^{S}\right)}{\left(\eta^{F}\right)^{2} \rho^{S}+\left(\eta^{S}\right)^{2} \rho^{F}}} .
$$

If the pore liquid is absent or gas is filled in the pores, then $\rho^{F}$ is very small as compare to $\rho^{S}$ and can be neglected. So the equation (24) reduces to

$$
c_{0}=\sqrt{\frac{\lambda^{S}+2 \mu^{S}}{\rho^{S}}} .
$$

This gives the velocity of the longitudinal wave propagating in an incompressible empty porous solid, where the change in volume is due to the change in porosity and is a well-known result of the classical theory of elasticity. In an incompressible non-porous solid medium $\eta^{F} \rightarrow 0$, then (24) yields $c_{1}=0$ and is physically acceptable as a longitudinal wave cannot propagate in an incompressible medium.

Equations (11) to (15) and (19) to (22), with the help of (23) reduce to the dimensionless form and the displacement components $u^{i}$ and $w^{i}(i=F, S, L)$ are expressed in terms of 
potential $\varphi^{i}, \psi^{i}$ and $\varphi^{L}$ as:

$$
\begin{aligned}
u^{i} & =\frac{\partial \varphi^{i}}{\partial x}+\frac{\partial \psi^{i}}{\partial z}, \\
w^{i} & =\frac{\partial \varphi^{i}}{\partial z}-\frac{\partial \psi^{i}}{\partial x}, \\
u^{L} & =\partial \varphi^{L} / \partial x \\
w^{L} & =\partial \varphi^{L} / \partial z
\end{aligned}
$$

and yield the following seven equations determining $\varphi^{S}, \varphi^{F}, \psi^{S}, \psi^{F}, p, \varphi^{L}$, and $p^{L}$ as:

$$
\begin{aligned}
& \nabla^{2} \varphi^{S}-\eta^{S} p-\delta_{2}^{2} \frac{\partial^{2} \varphi^{S}}{\partial t^{2}}+\delta_{2}^{2} P\left(\frac{\partial \varphi^{F}}{\partial t}-\frac{\partial \varphi^{S}}{\partial t}\right)=0, \\
& \varphi^{F}=-\frac{\eta^{S}}{\eta^{F}} \varphi^{S} \\
& \left(\eta^{F}\right)^{2} p-\delta_{3} \delta_{2}^{2} \eta^{S} \frac{\partial^{2} \varphi^{S}}{\partial t^{2}}-P \delta_{2}^{2} \frac{\partial \varphi^{S}}{\partial t}=0, \\
& \delta^{2} \nabla^{2} \psi^{S}-\delta_{2}^{2} \frac{\partial^{2} \psi^{S}}{\partial t^{2}}+P \delta_{2}^{2}\left(\frac{\partial \psi^{F}}{\partial t}-\frac{\partial \psi^{S}}{\partial t}\right)=0, \\
& \delta_{3} \frac{\partial^{2} \psi^{F}}{\partial t^{2}}+P\left(\frac{\partial \psi^{F}}{\partial t}-\frac{\partial \psi^{S}}{\partial t}\right)=0, \\
& \nabla^{2} \varphi^{L}=\delta_{6}^{2} \frac{\partial^{2} \varphi^{L}}{\partial t^{2}}, \\
& p^{L}=-\tau_{z z}^{L}=-\delta_{4} \delta_{5}^{2} \nabla^{2} \varphi^{L}
\end{aligned}
$$

where

$$
\begin{aligned}
& \delta=\frac{\beta_{0}}{c_{0}}, \quad \delta_{2}=\frac{c_{1}}{c_{0}}, \quad \beta_{0}=\sqrt{\frac{\mu^{S}}{\rho^{S}}}, \quad \delta_{3}=\frac{\rho^{F}}{\rho^{S}}, \quad P=\frac{S_{V}}{\omega^{\prime} \rho^{S}}, \quad \delta_{4}=\frac{\rho^{L}}{\rho^{S}}, \\
& \delta_{5}=\frac{\alpha_{L}}{c_{0}}, \quad \delta_{6}=\frac{c_{1}}{\alpha_{L}}, \quad \alpha_{L}=\sqrt{\frac{\lambda^{L}}{\rho^{L}}} .
\end{aligned}
$$

The boundary conditions are

$$
\begin{aligned}
& \tau_{z z}^{L}=0 \text { at } z=-H, \\
& w^{S}=w^{L} \text { at } z=0, \\
& \tau_{z z}-p=\tau_{z z}^{L} \text { at } z=0, \\
& \tau_{z x}=0 \text { at } z=0 .
\end{aligned}
$$


Equations (30) to (36) along with the conditions (38) to (41) govern the propagation of surface waves in an incompressible half-space lying under a uniform layer of a liquid. The time harmonic wave solutions of equations (30) to (35) for the wave propagation are given by

$$
\begin{aligned}
\varphi^{S} & =\left(P_{1} \cosh \xi_{1} z+P_{2} \sinh \xi_{1} z\right) e^{i k(x-c t)}, \\
\varphi^{F} & =-\frac{\eta^{S}}{\eta^{F}}\left(P_{1} \cosh \xi_{1} z+P_{2} \sinh \xi_{1} z\right) e^{i k(x-c t)}, \\
\psi^{S} & =\left(P_{3} \cosh \xi_{2} z+P_{4} \sinh \xi_{2} z\right) e^{i k(x-c t)}, \\
\psi^{F} & =\left(\frac{i P}{\delta_{3} k c+i P}\right)\left(P_{3} \cosh \xi_{2} z+P_{4} \sinh \xi_{2} z\right) e^{i k(x-c t)}, \\
\varphi^{L} & =\left(P_{5} \cosh \xi_{3} z+P_{6} \sinh \xi_{3} z\right) e^{i k(x-c t)},
\end{aligned}
$$

where

$$
\begin{aligned}
\xi_{1}^{2} & =k^{2}\left(1-c^{2}-i \frac{Q c}{k}\right) \\
\xi_{2}^{2} & =k^{2}\left\{1-\delta_{2}^{2}\left(1+i \frac{\delta_{3} P}{\left(k c \delta_{3}+i P\right)}\right)\left(\frac{c}{\delta}\right)^{2}\right\} \\
\xi_{3}^{2} & =k^{2}\left(1-\delta_{6}^{2} c^{2}\right) \\
Q & =\frac{P \delta_{2}^{2}}{\left(\eta^{F}\right)^{2}}
\end{aligned}
$$

From equations (26) to (29), (36), along with the stress-strain relations (6) and with the help of (42) to (46), we obtain the following expressions for displacements, pore pressure and stresses as:

$$
\begin{aligned}
u^{S}= & \left\{i k\left(P_{1} \cosh \xi_{1} z+P_{2} \sinh \xi_{1} z\right)+\xi_{2}\left(P_{3} \sinh \xi_{2} z+P_{4} \cosh \xi_{2} z\right)\right\} e^{i k}(x-c t), \\
w^{S}= & \left\{\xi_{1}\left(P_{1} \sinh \xi_{1} z+P_{2} \cosh \xi_{1} z\right)-i k\left(P_{3} \cosh \xi_{2} z+P_{4} \sinh \xi_{2} z\right)\right\} e^{i k}(x-c t), \\
u^{F}= & {\left[-\frac{\eta^{S}}{\eta^{F}} i k\left(P_{1} \cosh \xi_{1} z+P_{2} \sinh \xi_{1} z\right)\right.} \\
& \left.+\frac{i P \xi_{2}}{\delta_{3} k c+i P}\left(P_{3} \sinh \xi_{2} z+P_{4} \cosh \xi_{2} z\right)\right] e^{i k}(x-c t), \\
w^{F}= & {\left[-\frac{\eta^{S}}{\eta^{F}} \xi_{1}\left(P_{1} \sinh \xi_{1} z+P_{2} \cosh \xi_{1} z\right)\right.} \\
& \left.+\frac{k P}{\delta_{3} k c+i P}\left(P_{3} \cosh \xi_{2} z+P_{4} \sinh \xi_{2} z\right)\right] e^{i k}(x-c t),
\end{aligned}
$$




$$
\begin{aligned}
u^{L}= & i k\left(P_{5} \cosh \xi_{3} z+P_{6} \sinh \xi_{3} z\right) e^{i k}(x-c t) \\
w^{L}= & \xi_{3}\left(P_{5} \sinh \xi_{3} z+P_{6} \cosh \xi_{3} z\right) e^{i k}(x-c t) \\
p= & -\frac{\delta_{2}^{2}}{\left(\eta^{F}\right)^{2}}\left(k^{2} c^{2} \eta^{S} \delta_{3}+i P k c\right)\left(P_{1} \cosh \xi_{1} z+P_{2} \sinh \xi_{1} z\right) e^{i k}(x-c t) \\
\tau_{z z}= & {\left[\left\{\xi_{1}^{2}-\left(1-2 \delta^{2}\right) k^{2}\right\}\left(P_{1} \cosh \xi_{1} z+P_{2} \sinh \xi_{1} z\right)\right.} \\
& \left.-2 \delta^{2} i k \xi_{2}\left(P_{4} \cosh \xi_{2} z+P_{3} \sinh \xi_{2} z\right)\right] e^{i k}(x-c t) \\
\tau_{x z}= & \delta^{2}\left\{2 i k \xi_{1}\left(P_{1} \sinh \xi_{1} z+P_{2} \cosh \xi_{1} z\right)\right. \\
& \left.+\left(k^{2}+\xi_{2}^{2}\right)\left(P_{3} \cosh \xi_{2} z+P_{4} \sinh \xi_{2} z\right)\right\} e^{i k}(x-c t) \\
\tau_{z z}^{L}= & \delta_{4} \delta_{5}^{2}\left(\xi_{3}^{2}-k^{2}\right)\left(P_{5} \cosh \xi_{3} z+P_{6} \sinh \xi_{3} z\right) e^{i k}(x-c t)
\end{aligned}
$$

Substituting the equations (51) to (60) in the boundary conditions (38) to (41) and in order to insure the boundedness of solutions at infinity, one can easily obtain the following system of linear simultaneous equations for the unknown arbitrary constants $P_{1}, P_{2}, P_{3}, P_{4}, P_{5}$, and $P_{6}$ as:

$$
\begin{aligned}
& P_{1}+P_{2}=0 \\
& P_{3}+P_{4}=0 \\
& P_{5} \cosh \xi_{3} H-P_{6} \sinh \xi_{3} H=0 \\
& 2 i k \xi_{1} P_{2}+\left(k^{2}+\xi_{2}^{2}\right) P_{3}=0 \\
& R P_{1}-2 \delta^{2} i k \xi_{2} P_{4}-S P_{5}=0 \\
& \xi_{1} P_{2}-i k P_{3}-\xi_{3} P_{6}=0
\end{aligned}
$$

where

$$
R=\delta^{2} k^{2}\left[2-\left\{1-\frac{\delta_{2}^{2} \eta^{S} \delta_{3}}{\left(\eta^{F}\right)^{2}}\right\}\left(\frac{c}{\delta}\right)^{2}\right] \text { and } S=\delta_{4} \delta_{5}^{2}\left(\xi_{3}^{2}-k^{2}\right) .
$$

The non-trivial solution of above system of equations requires that the determinant of the coefficients of the unknown must vanish. This argument yields the required frequency equation relating the non-dimensional phase velocity $c$ with the non-dimensional wave number $k$ as:

$$
\tanh \xi_{3} H=\frac{\xi_{3}\left\{\left(\xi_{2}^{2}+k^{2}\right)\left(2 \delta^{2} \xi_{1} \xi_{2}-R\right)-2 \delta^{2} \xi_{1} \xi_{2}\left(\xi_{2}^{2}-k^{2}\right)\right\}}{\xi_{1} S\left(\xi_{2}^{2}-k^{2}\right)} .
$$

\section{A particular case}

Rayleigh type surface waves at the free surface of a fluid-saturated incompressible porous half-space: 
If $H=0$ then after some simplifications, equation (68) takes the form

$$
\begin{aligned}
& \sqrt[4]{1-c^{2}-i \frac{Q c}{k}} \sqrt{1-\delta_{2}^{2}\left(1+i \frac{\delta_{3} P}{k c \delta_{3}+i P}\right)\left(\frac{c}{\delta}\right)^{2}} \\
& =\left\{2-\delta_{2}^{2}\left(1+i \frac{\delta_{3} P}{k c \delta_{3}+i P}\right)\left(\frac{c}{\delta}\right)^{2}\right\}\left[2-\left\{1-\frac{\delta_{2}^{2} \eta^{S} \delta_{3}}{\left(\eta^{F}\right)^{2}}\right\}\left(\frac{c}{\delta}\right)^{2}\right] .
\end{aligned}
$$

Equation (69) governs the propagation of Rayleigh type surface waves at the free surface of a fluid-saturated incompressible porous half-space and if the pore liquid is absent or gas is filled in the pores then after some simplifications equation (69) takes the form

$$
\sqrt[4]{\left(1-\frac{c^{2}}{\delta^{2}}\right)\left(1-c^{2}\right)}=\left(2-\frac{c^{2}}{\delta^{2}}\right)^{2}
$$

If the dimensionless quantities are converted in to the corresponding physical quantities then this equation yields

$$
\sqrt[4]{\left(1-\frac{c^{2}}{c_{0}^{2}}\right)\left(1-\frac{c^{2}}{\beta_{0}^{2}}\right)}=\left(2-\frac{c^{2}}{\beta_{0}^{2}}\right)^{2}
$$

The above equation corresponds to the Raleigh wave propagating in the empty incompressible porous solid and is a well-known result of classical theory.

Now, we derive the equations of the trajectory of particle motion during the propagation Rayleigh type waves in a fluid-saturated incompressible porous half-space. The horizontal and vertical components of displacement of a solid particle are real valued and are provided by equations (51) and (52). So, after some simplifications the trajectory of a solid particle at a depth $\mathrm{z}_{0}$ is defined by

$$
\frac{\left(u^{S}\right)^{2}}{a^{2}}+\frac{\left(w^{S}\right)^{2}}{b^{2}}=1
$$

Therefore, like the classical theories, trajectory of solid particle motion in this case is also elliptic with the axes ' $a$ ' and ' $b$ ' as the real parts of the quantities respectively and are different from their corresponding values as given in the classical theories.

$$
k P_{1}\left(e^{-\xi_{1} z_{0}}-\frac{2 \xi_{1} \xi_{2}}{\xi_{2}^{2}+k^{2}} e^{-\xi_{2} z_{0}}\right) \text { and }-\xi_{1} P_{1}\left(e^{-\xi_{1} z_{0}}-\frac{k^{2}}{\xi_{2}^{2}+k^{2}} e^{-\xi_{2} z_{0}}\right)
$$

The vertical motion vanishes at a depth $H_{0}$ given by

$$
H_{0}=\frac{1}{\xi_{1}-\xi_{2}} \log \left(\frac{2 \delta^{2} \xi_{1} \xi_{2}}{R}\right)
$$

The ellipse will then stretched out into a straight line, $u^{S}=a$, and will alter its direction beyond this depth. 


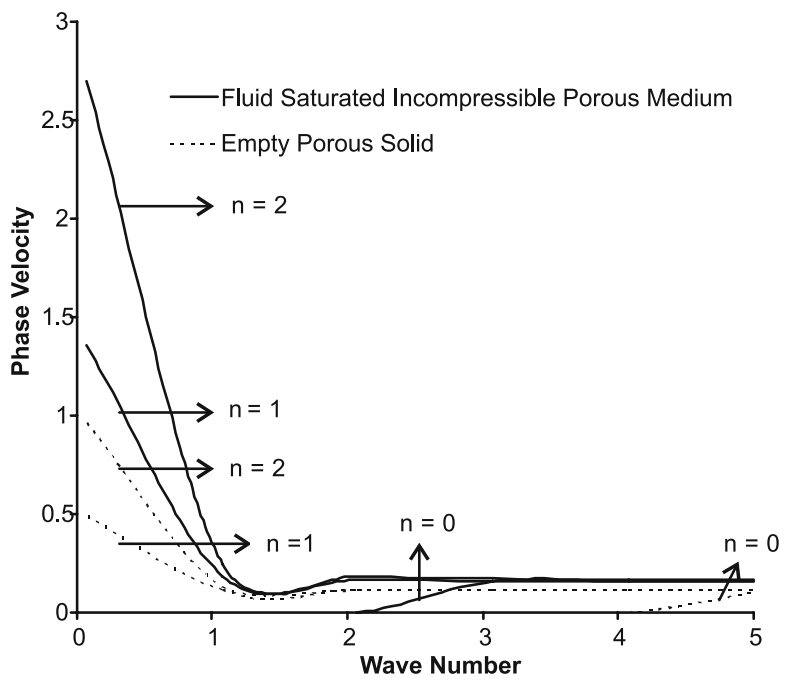

Figure 2. Variation of phase velocity with wave number.

\section{Discussion}

Following de Boer et al (1993) and Ewing et al (1957), the values of various physical parameters are taken as $\eta^{S}=0.67, \eta^{F}=0.33, \rho^{S}=1.34 \mathrm{Mg} / \mathrm{m}^{3}, \rho^{F}=0.33 \mathrm{Mg} / \mathrm{m}^{3}, \lambda^{S}=$ $5.5833 \mathrm{MN} / \mathrm{m}^{2}, \mu^{S}=8.3750 \mathrm{MN} / \mathrm{m}^{2}, k^{F}=0.01 \mathrm{~m} / \mathrm{s}, \gamma^{F R}=10.00 \mathrm{kN} / \mathrm{m}^{3}, \alpha^{L}=1.463 \times$ $10^{3} \mathrm{~m} / \mathrm{s}$. The variation of non-dimensional phase velocity and attenuation coefficient with respect to non-dimensional wave number is graphically presented in figures 2 and 3 . As $\tanh (i n \pi+z)=\tanh z$, so for given values of wave number, equation (68) is solved for different integral values of $n$ and for a fix value of $H$. For $n=0$, the phase velocity increases uniformly from a very small value and then becomes constant indicating that the wave for the

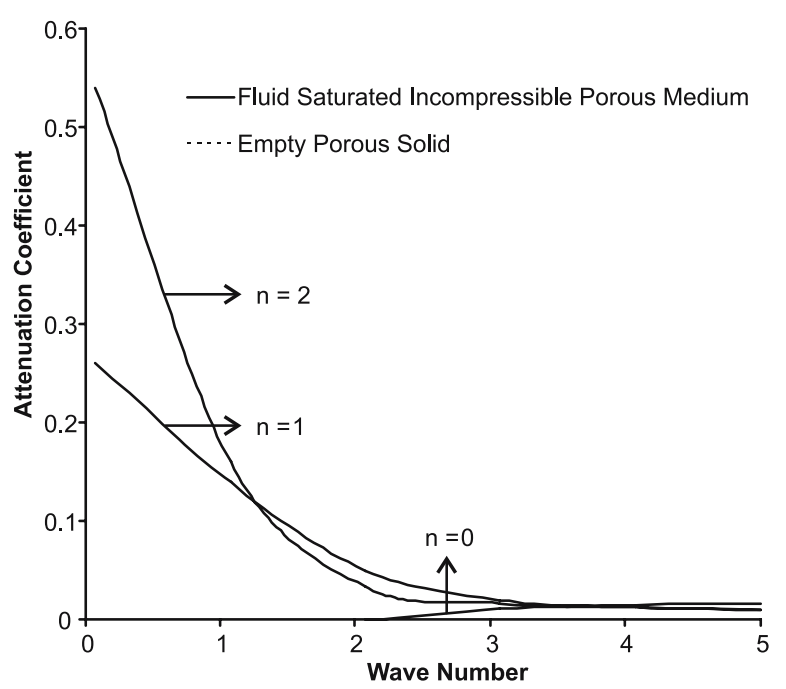

Figure 3. Variation of attenuation coefficient with wave number. 
first mode is non-dispersive. But, for all other values of $n$, it is evident that the phase velocity depends upon the wave number, showing that the waves for all other modes are dispersive. Here for any value of $n$, starting from some maximum value, the phase velocity decreases very quickly to some small value, then increases and ultimately becomes constant. It indicates that for small values of wave number, the wave is highly dispersive, but its dispersiveness decreases with the increase of wave number and ultimately it becomes non-dispersive. If the pore liquid is absent or gas is filled in the pores, then $\rho^{F}$ is very small as compared to $\rho^{S}$ and hence it can be neglected. This situation for empty porous solid is represented by dotted lines in the graphs. The behaviour of the dispersion curves in both cases is almost similar, but for any value of $n$, the curve for empty porous solid lies below the corresponding curve for fluid-saturated medium indicating that the presence of fluid in the pores increases the phase velocity. Figure 3 represents the variation of attenuation coefficient with that of nondimensional wave number. For the first mode, the attenuation coefficient is almost zero and for all other modes, starting from some higher value, the attenuation coefficient decreases gradually with wave number and ultimately becomes zero. As far as the empty porous solid is concerned, the attenuation coefficients are zero for all modes. Another important property of the surface waves is that, the phase velocity for both the cases and the attenuation coefficient for fluid-saturated incompressible porous medium increase with $n$ for the lower values of the wave number, but for large values of the wave number both these quantities become constant and equal for all values of $n$.

\section{Conclusion}

The propagation of surface waves in a fluid-saturated incompressible porous half-space lying under a uniform layer of a liquid is discussed. The half-space is modelled as a two-phase system with two incompressible constituents (porous solid and inviscid fluid), where the general field equations are directly adopted according to de Boer \& Ehlers (1990). A layer of water is taken for numerical investigation and during the investigation of the properties of the dispersion relation; it is observed that the first mode is non-dispersive, whereas all other modes propagate dispersively. Rayleigh type surface waves at the free surface of an incompressible porous half-space are also discussed and the results, at various steps, are compared with the classical theories.

\section{References}

Achenbach J D (1976) Wave propagation in elastic solids. (Amsterdam: North-Holland Publishing Company)

Auriault J L (1980) Dynamic behaviour of a porous medium saturated by a newtonian fluid. Int. J. Eng. Sci. 18: 775-785

Biot M A (1941) General theory of three dimensional consolidation. J. Appl. Phys. 12: 155-161

Biot M A (1956a) Theory of propagation of elastic waves in a fluid-saturated porous solid - I. Low frequency range. J. Acout. Soc. Am. 28: 168-178

Biot M A (1956b) Theory of propagation of elastic waves in a fluid-saturated porous solid - II. Higher frequency range. J. Acout. Soc. Am. 28: 179-191

Biot M A (1962) Mechanics of deformation and acoustic propagation in porous media. J. Appl. Phys. 33: $1482-1498$

Bowen R M (1980) Incompressible porous media models by use of the theory of mixtures. Int. J. Eng. Sci. 18: 1129-1148 
de Boer R, Ehlers W (1990) The development of the concept of effective stress theories. Acta Mech. 83: 77-92

de Boer R, Ehlers W (1990) Uplift, friction and capillarity - Three fundamental effects for liquidsaturated porous solids. Int. J. Solids Struct. 26: 43-57

de Boer R, Ehlers W, Liu Z (1993) One-dimensional transient wave propagation in a fluid-saturated incompressible porous media. Arch. Appl. Mech. 63: 59-72

de Boer R, Ehlers W (1988) A historical review of the formulation of porous media theories. Acta Mech. 74: 1-8

de Boer R (2000) Theory of porous media. (New York: Springer-Verleg)

Edelman I (2004) Surface waves at vacuum/porous medium interface: low frequency range. Wave Motion 39: 111-127

Ehlers W (1993) Compressible, incompressible and hybrid two-phase models in porous theories. ASME; AMD 158: 25-38

Ewing W M, Jardetzky W S, Press F (1957) Elastic waves in layered media, (: Mc Graw Hill)

Fillunger P (1933) Der auftrieb in talsperren. osterr. wochenschrift fur den offentl. baudienst [M]. I. Teil 532-552, II. Teil 552-556, III. Teil 567-570.

Kumar R, Miglani A (1996) Effect of pore alignment on surface wave propagation in a liquid-saturated porous layer over a liquid-saturated porous half-space with loosely bonded interface. J. Phys. Earth 44: $1317-1337$

Kumar R, Deswal S (1996) Surface wave propagation in liquid-saturated porous layer over a liquidsaturated porous half-space with loosely bonded interface. J. Phys. Earth, 44: 1317-1337

Kumar S, Hundal B S (2002) A Study of spherical and cylindrical wave propagation in a nonhomogeneous fluid-saturated incompressible porous medium by method of characteristics. current trends in industrial and applied mathematics. Ed. P Manchanda et al New Delhi: Anamya Publisher 181-194

Kumar R, Hundal B S (2003) Wave propagation in a fluid-saturated incompressible porous medium. Indian J. Pure Appl. Math. 4: 651-665

Kumar R, Hundal B S (2003) One-dimensional wave propagation in a non-homogeneous fluidsaturated incompressible porous medium. Bull. Allahabad Math. Soc. 18: 1-13

Kumar R, Hundal B S (2004) Effect of non-homogeneity on one-dimensional wave propagation in a fluid-saturated incompressible porous medium. Bull. Callif. Math. Soc. 96: 179-188

Kumar R, Hundal B S (2004) Symmetric wave propagation in a fluid-saturated incompressible porous medium. J. Sound Vib. 96: 179-188

Levy T (1979) Propagation of waves in a fluid-saturated porous elastic solids. Int. J. Eng. Sci. 17: $1005-1014$

Liu K, Liu Y (2004) Propagation characteristics of rayleigh waves in orthotropic fluid-saturated porous media. J. Sound Vib. 271: 1-13

Prevost J H (1982) Nonlinear transient phenomena in saturated porous media. Comp. Math. Appl. Mech. Eng. 3-18

von Terzaghi K (1923) Die Berechnug der Durchlassigkeit des Tones aus dem Verlauf der hydromechanischen Spannungserscheinungen. Sitzungsber. Akad. Wiss. (Wien), Math. Naturwiss. Kl., Abt. IIa 132: $125-138$

von Terzaghi K (1925) Erdbaumechanik auf Bodenphysikalischer Grundlage, p. 399. Leipzig - wien: Franz Deuticke

Zienkiewicz O C, Shiomi T (1984) Dynamic behaviour of saturated porous media - the generalized biot formulation and its numerical solution. Int. J. Numer. Ana. Methods Geomech. 8: 71-96 